sciendo

\title{
Ceteris Paribus Laws and the Human Sciences
}

\author{
Rui Silva \\ University of the Azores and LanCog
}

Disputatio Vol. 4, No. 34

December 2012

DOI: $10.2478 /$ disp-2012-0034

ISSN: 0873-626X 


\title{
Ceteris Paribus Laws and the Human Sciences
}

\author{
Rui Silva \\ University of the Azores and LanCog Group \\ BIBLID [0873-626X (2012) 34; pp. 851-867]
}

In opposition to the traditional conception of scientific laws as universal and exceptionless generalizations that hold everywhere and throughout all times, some philosophers of science have proposed the idea of a ceteris paribus law (CP law), i.e., a law that is true "all else being equal", in the sense that it can admit of exceptions because of the influence of interfering factors. The legitimacy of CP laws has divided the philosophical community. Some argue that all scientific laws are $\mathrm{CP}$ laws, others argue that $\mathrm{CP}$ laws are simply unacceptable, and a third group claims that science contains both CP laws (in the so-called special sciences) and strict laws (in basic physics).

The debate on CP laws has important implications for our understanding of the human sciences, because if we accept the existence of laws in sciences like psychology or sociology, these laws, far from being strict, should be CP laws. Furthermore, if we could establish some differences between CP laws in the human and the natural sciences, such a difference could help us to understand the relation between these two scientific domains.

In what follows, I will first discuss the concept of CP law and present some proponents of such laws (1). In order to explore the significance of this debate for the human sciences, I will discuss Davidson's and Fodor's stance on the status of CP laws in the human sciences (2). After these introductory sections, I will argue that in the human sciences it is much more difficult, not only in practice, but also in principle, to cope with interfering factors and to refine ceteris paribus clauses (3). Against hermeneutic and strict naturalistic perspectives, I propose an account of the human sciences that rec-

Disputatio, Vol. IV, No. 34, December 2012 
ognizes the methodological role of both generalizations and rationality assumptions; in particular, I will stress the need for interpretative/hermeneutic methods to control the vagueness of ceteris paribus clauses (4).

A fundamental problem in the philosophy of the human sciences consists in the existence or possibility of laws in these sciences. Because of the complexity of the factors that can influence human behaviour, generalizations in psychology and sociology, for instance, have a somewhat fragile status, especially when they are couched in an intentional vocabulary. Predictions based on such generalizations may well fail, and it is for this reason that the idea of CP laws, understood as laws that admit of exceptions, becomes relevant for understanding the human sciences. ${ }^{1}$

A CP law describes real tendencies or causal powers, but it idealizes the phenomena to be explained and abstracts them from the possible interference of factors that lie beyond its scope. It is in this sense that CP laws admit of exceptions and can be contrasted with strict laws. According to the traditional conception, scientific laws are universally valid, empirically confirmable and counterfactualsupporting generalizations. They can be either strict or statistic, but in both cases they are conceived as exceptionless. A CP law, on the other hand, is a generalization that is true "all else being equal", i.e., exceptions are possible because of the interference of an indefinite number of factors that cannot be specified in advance.

The idea of CP laws is, of course, controversial. In the first place, these laws seem to be vacuous. Let us consider the following formulation of a law with a ceteris paribus clause: "All As are Bs, except when they are not". This is a caricature of a CP law, but it points to a real problem with $\mathrm{CP}$ laws, its apparent vacuity. Closely related to this objection is the charge of vagueness. CP laws are true all else being equal. But what is exactly "all else" (ceteris)? And what is the exact

\footnotetext{
${ }^{1}$ Ceteris paribus laws have already been labelled as soft laws (cf. Horgan/Tienson 1996), and they are, as such, appropriate candidates for the role of laws of the so-called soft sciences.
} 
meaning of "being equal" (paribus) in a particular context? If CP laws lack determinate truth conditions, advocates of truth-conditional theories of meaning can doubt that they have meaning.

Last, but not least, CP laws seem to evade the requirements of testability or falsifiability. The idea that laws may have exceptions because of the interference of unexpected factors threatens to immunize them against empirical refutation; it allows the scientist to hold a law in the light of recalcitrant evidence by introducing ad hoc hypotheses. ${ }^{2}$

However, and according to their proponents, CP laws are legitimate. To begin with, there are many scientific generalizations that have exceptions but are considered as genuine laws; Boyle's law and the law of supply and demand are two good examples. The supporters of CP laws also claim that, in order to study a very complex world, science has inevitably to appeal to abstractions and idealizations. CP laws belong to these idealizations; they single out causal tendencies that, far from operating in isolation, interact with many other causal tendencies. Since events result from a combination of causes, a real tendency may be overridden by other causal forces, and this would entitle us to introduce ceteris paribus clauses in lawstatements.

Carl Hempel's reflections on the role of provisos was a major source of inspiration for supporters of CP laws. He claimed that Newton's law of gravitation involves certain assumptions and can lead to inaccurate predictions if, for instance, non-gravitational forces interfere with gravitational ones. As a result, predictions based on Newton's law are true, provided that no significant external forces (gravitational or non-gravitational ones) affect a planetary system. Since an indefinite number of forces may act on the planets, the proviso seems to have an open-ended character similar to ceteris paribus clauses. For this reason, some authors attributed to Hempel the thesis that even the fundamental laws of physics contain ceteris paribus

\footnotetext{
${ }^{2}$ In the words of Roberts (2004: 162): "hedged regularities cannot be discovered by science, because they are not empirically testable. The hedge functions as an 'escape clause' that allows any hypothesized hedged regularity to escape empirical refutation: Whenever you discover a counterexample, claim that there has been an interference of some kind, so that the case is outside the scope of the hypothesis and does not falsify it".
} 
clauses (cf., e.g., Fodor 1991). This interpretation of Hempel's conception of provisos is, however, dubious. Earman and Roberts (1999: 442-446) have argued, in my view persuasively, that the conditions of the Hempelian provisos are not relative to the truth of law statements, but only to the validity of the application of a theory. This point distinguishes his provisos from current accounts of ceteris paribus clauses.

Another important voice in the debate on CP laws is Nancy Cartwright. She was known for a long time as a supporter of $\mathrm{CP}$ laws because of her opposition to a Humean understanding of laws as regularities and her thesis that scientific laws should be understood in the "language of powers, capacities or natures and related concepts such as interfere, inhibit, facilitate, and trigger" (Cartwright 2002: 150). She has, indeed, claimed that scientific laws are CP laws in the sense that "they hold only relative to the successful repeated operation of a nomological machine” (Cartwright 1997: 66). By nomological machine she understands a set of capacities that cause in a stable environment the regularities that are described by scientific laws. Nomological machines are also described in her work as the object of scientific models. $^{3}$

Fodor (1991) and Pietroski/Rey (1995) represent another influential defence of $\mathrm{CP}$ laws. In spite of some differences, their accounts of $\mathrm{CP}$ laws share some common assumptions, namely that scientific explanations rely on laws and that strict laws must be exceptionless. Since many scientific generalizations admit of exceptions, they claim that for a given CP law there is a condition that, when realized, eliminates exceptions. Fodor calls such a condition a "completer". The combination of a CP law with the corresponding completer amounts to a strict or exceptionless law. In the case of Pietrosky and Rey, $\mathrm{CP}$ laws are considered genuine laws if prediction failures can be explained by a fact that has an independent explanatory role. More precisely, an exception to a scientific law is legitimate if we can indicate an interfering factor and if this factor can be explained on the

\footnotetext{
${ }^{3}$ More recently, Cartwright has distanced herself from CP laws, but the point is apparently terminological; she does not agree with characterizations of CP laws as laws that neither can be stated in precise and closed form nor entail strict or statistical regularities (cf. Cartwright 2002: 149). At any rate, she remains faithful to an understanding of laws in terms of powers and capacities.
} 
basis of an independent theory. This condition allows us to prevent ad hoc manoeuvres in science. Pietroski and Rey illustrate this point in the following metaphorical terms:

But there need be no intrinsic problem about ineliminable CP-clauses. Briefly, we claim that such clauses are cheques written on the bank of independent theories, their substance and warrant deriving from the substance and warrant of those theories, which determine whether the cheque can be cashed." (Pietroski/Rey 1995: 82)

This approach has the advantage of defending CP laws from the charge of vacuity without trying to give determinate truth conditions to them.

At a more methodological level, there are some elaborate attempts to establish the legitimacy of CP laws. A case in point is Harold Kincaid. In chapter 3 of his Philosophical Foundations of the Social Sciences, he claims that, appearances notwithstanding, CP laws are serious and testable laws. It would be inappropriate in this context to delve into his reflections on the testability problem, but one can highlight some of his main points. He stresses, for instance, that real situations fit sometimes scientific idealizations, and in these cases $\mathrm{CP}$ laws become clearly testable. Furthermore, exceptions can be often explained away by reference to well-established laws or causal mechanisms, and successive refinements may increase the accuracy of the predictions. Kincaid also stresses that $\mathrm{CP}$ laws may predict novel facts and support counterfactuals.

He cites in this context the work of the sociologist Jeffrey Paige on the relations between agrarian structure and political behaviour as an example of good social science that offers us lawlike generalizations (Kincaid 1996: 70-80). By gathering the relevant evidence, Paige formulated a set of interesting generalizations that do not express strict regularities, but have other traits of the traditional conception of law; they are, namely, empirically confirmable and counterfactual-supporting. Here are some examples of these generalizations:

1. Plantation systems (where owners depend on capital and workers on wages) encourage collective action and labor reforms.

2. Commercial hacienda systems (where both owners and cul- 
tivators get their income from land) favour agrarian revolts.

3. Large estate systems (where owners get their income from the land and cultivators from wages) tend to lead to revolutionary movements.

Paige was able to confront these generalizations with the available data and concluded that they hold generally. The generalizations include, of course, ceteris paribus clauses, but one of the merits of his work consists precisely in showing how a researcher can refine the ceteris paribus clauses by considering the relevant interfering factors and by explaining their influence on the causal tendencies expressed by the generalizations. For instance, he shows how contagion effects (the increased probability of an event on the basis of previous occurrences of similar events) and the action of urban political parties influence the political behaviour of cultivators.

What can we conclude from the preceding considerations? It would be too ambitious to try to settle here the intricate debate on the legitimacy of CP laws. ${ }^{4}$ For the purposes of the present article, it is sufficient to clarify the concept of $\mathrm{CP}$ law, to understand the motivation behind it, to evaluate the significance of scientific generalizations with ceteris paribus clauses and to compare their status in the natural and in the human sciences. In fact, even if one refuses to accept CP laws as genuine laws, one may well accept that there are many scientific generalizations that admit of exceptions, but are testable and important for science. ${ }^{5}$

As we have seen, many respectable lawlike statements must inevitably idealize phenomena that, in the real world, are the result of very complex causal interactions that cannot be grasped by unqualified universal statements. Lawlike generalizations typically describe

${ }^{4}$ For a good survey on the main accounts of CP laws and their difficulties, see Earman/Roberts 1999.

${ }^{5} \mathrm{~A}$ case in point is Jim Woodward, who denies the existence of CP laws, but accepts the corresponding generalizations: "While I reject the idea that generalizations found in the special sciences are ceteris paribus laws, I fully agree that many of those generalizations are 'scientifically legitimate', that they are testable and in fact strongly supported by evidence [...]. My claim is that construing those generalizations as ceteris paribus laws is the wrong way to defend their usefulness and legitimacy" (Woodward 2002: 306). 
a causal tendency, but a tendency that interacts in the real world with other tendencies. This lends to generalizations with ceteris paribus clauses a prima facie legitimacy. However, we should keep in mind that the existence of ceteris paribus clauses weakens a nomological statement and may undermine its explanatory power.

\section{2}

In order to evaluate the significance of CP laws for the human sciences it is convenient to consider the work of Davidson and Fodor in this context. Both of them accept the existence of CP laws in the special sciences, but evaluate differently their role in the human sciences.

Davidson argued in a series of influential papers that there are no strict psychological laws. By strict law he understands the traditional conception of law as a non-accidental generalization, characterized by being empirically confirmable and by supporting counterfactuals. Intentional psychology produces many useful generalizations, but, according to Davidson, they are not strict laws; far from being exceptionless, they are vulnerable to the interference of an indefinite number of factors that cannot be specified in advance.

His defence of the Principle of the Anomalism of the Mental is based on a rejection of psychophysical laws. The basic point behind psychophysical anomalism is the claim that the vocabularies of physics and psychology are incommensurable:

There are no strict psychophysical laws because of the disparate commitments of the mental and physical schemes. It is a feature of physical reality that physical change can be explained by laws that connect it with other changes and conditions physically described. It is a feature of the mental that the attribution of mental phenomena must be responsible to the background of reasons, beliefs, and intentions of the individual. (Davidson 1980: 222)

According to Davidson, the gap between the vocabularies of physics and psychology results from the holistic and normative character of the psychological domain. The content of mental states is partly determined by their reciprocal relations and its interpretation is based on rationality assumptions:

Any effort at increasing the accuracy and power of a theory of behaviour forces us to bring more and more of the whole system of the agent's beliefs and motives directly into account. But in inferring this 
system from the evidence, we necessarily impose conditions of coherence, rationality, and consistency. These conditions have no echo in physical theory, which is why we can look for no more than rough correlations between psychological and physical phenomena" (Davidson 1980: 231)

Another key point of the Davidsonian defence of the anomalism of the mental departs from the idea that a strict or exceptionless law requires a closed domain, like the domain of physics. In order to explain physical phenomena we do not need to accommodate the possible interference of non-physical causes; on the contrary, physicists and philosophers of science endorse the principle of the causal closure of physics. But the psychological domain is clearly not closed. Psychological phenomena are exposed to the action of non-psychological causes; biological, chemical and physical factors. For this reason, we cannot formulate a psychological theory that provides a complete coverage of the psychological domain. Psychological anomalism, the thesis that there are no strict laws describing correlations between psychological phenomena, can be presented as a consequence of the conjunction of psychophysical anomalism and the claim that the psychological domain is not closed (cf. Davidson 1980: 224).

Davidson is aware that scientific laws may admit exceptions, but he claims that in the natural sciences it is possible, in principle, to refine and improve laws in order to eliminate exceptions. Psychological generalizations are not refinable in this sense. Thus, in "Psychology as Philosophy" he clearly dismisses the possibility of strict laws dealing with beliefs and desires, the kind of laws that could ground intentional explanations of the human behaviour. In his perspective, it is hopeless to expect laws of the type "whenever a man has suchand-such beliefs and desires and such-and-such further conditions are satisfied, he will act in such-and such a way" (Davidson 1980: 233). He claims that:

What is needed in the case of action, if we are to predict on the basis of beliefs and desires, is a quantitative calculus that brings all relevant beliefs and desires into the picture. There is no hope of refining the simple pattern of explanation on the basis of reasons into such a calculus. (Davidson 1980: 233)

The lack of precision of psychological laws cannot be explained in the same way that we explain the lack of precision of some natural 
scientific laws; the laws involved in meteorological explanations and forecasts, for instance. The problem with psychological generalizations does not consist simply in the complexity of the factors that intervene in the human behaviour. Meteorological forecasts are admittedly fallible because of the complexity of meteorological phenomena, and earthquakes, according to the current status of seismology, cannot be predicted. Serious laws may have ceteris paribus clauses in order to accommodate the possible interference of factors that generate exceptions to them, but Davidson thinks that there is, at any rate, an important distinction between the ceteris paribus clauses of natural scientific laws and the corresponding clauses in putative psychological laws. In the former case, but not in the latter, it is possible, in principle, "to determine in advance whether or not the conditions of application are satisfied" (Davidson 1980: 233). This claim should be understood in the light of Davidson's holistic and normative conception of the psychological domain. Because different individuals have different sets of beliefs and desires, generalizations couched in an intentional vocabulary express mere tendencies and cannot aspire to the degree of precision that characterizes natural scientific laws. Furthermore, any attempt to explain and predict actions on the basis of beliefs and desires must rely, according to Davidson's Principle of Charity, on rationality assumptions, and these assumptions are not rigid; there are different ways to rationalize an agent's behaviour.

Davidson's defence of psychological anomalism is based on important insights, but is not satisfactorily developed in his work. In particular, he does not clarify the key notion of ceteris paribus law, although he appeals to laws of this kind, namely when he argues for the causal efficacy of mental events. In "Thinking Causes", Davidson appeals to non-strict laws in order to save his anomalous monism from the charge of epiphenomenalism. In fact, according to Davidson's principle that causal relations are covered by laws, he seems to be forced to appeal to psychophysical laws if he wants to attribute causal powers to mental states, but since strict laws cannot be couched in a mental vocabulary, laws that correlate mental events with physical events should be non-strict laws. A further advantage of these laws consists in the fact that they do not entail a physical reduction of the 
mental. ${ }^{6}$ Non-strict laws have, according to Davidson, the merit of explaining the causal efficacy of the mental while avoiding at the same time the threat of reductionism.

Fodor claimed, in opposition to Davidson, that the status of psychological generalizations does not differ from the status of many other laws in the natural sciences. Fodor concedes that intentional laws are non-strict laws, but does not think that this point raises a particular epistemological problem for psychology, because sciences like biology, meteorology or geology rely also on non-strict laws. Nomological generalizations in the so-called special sciences admit of exceptions, because of the presence of interfering factors that cannot be taken into account in the formulation of the law, but it is usually possible to explain exceptions to a law in the vocabulary of another, more basic sciences. In the case of psychology, exceptions to generalizations could be explained with the resources of sciences like neurology or biochemistry:

Exceptions to the generalizations of a special science are typically inexplicable from the point of view of (that is, in the vocabulary of) that science. That's one of the things that make it a special science. But, of course, it may it may nevertheless be perfectly possible to explain the exceptions in the vocabulary of some other science. (Fodor 1987: 6)

Fodor argues that the same holds for commonsense psychology; the ceteris paribus clauses of its generalizations are "ineliminable from the point of view of its proprietary conceptual resources", but "can be discharged in the vocabulary of some lower-level science" (Fodor 1987: 6).

Against Fodor, I argue that there are significant differences between psychological generalizations and laws of natural science. CP laws are prima facie legitimate because of the existence of interference effects

\footnotetext{
${ }^{6}$ Davidson was sensitive, in his context, to Kim's following objection: "The trouble is that once we begin talking about correlations and dependencies between specific psychological and physical properties, we are in effect talking about psycho-physical laws, and these laws raise the spectre of unwanted physical reductionism. Where there are psycho-physical laws, there is always the threat, or promise, of psycho-physical reduction.” (Kim 1993: 278-9).
} 
between different causal factors, but we must distinguish between two different types of interferences. The first type is what I will call external interference, and it is present whenever an exception to a generalization is caused by factors that belong to another domain, which is covered by another theory or science. It is dubious that this kind of interference has the same form in psychology and in natural sciences like biology or geology. In fact, psychological generalizations cannot be linked to, say, neurology in the same way that biological processes, for instance, can be linked to chemical processes. Psychological entities are partly constituted, as Davidson argued, on the basis of rationality assumptions and normative principles, and this sets them apart from the world of natural phenomena. By studying the chemical basis of a biological phenomenon one may understand it better, but neurological studies do not contribute to our understanding of reasons. McDowell's distinction between the "space of reasons" and the "realm of laws" may clarify this point. Those who argue that the relation between psychology and the corresponding lower-level theories (such as biology or chemistry) is analogous to the relation between higher-level and lower-level theories in the natural sciences neglect the fact that in the former case we are in presence of two different forms of intelligibility; in the latter case, we are dealing with different levels in the same domain (the "realm of law"). This means that in psychology and other human sciences the phenomena of external interference are much more difficult to handle (not only in practice, but also in principle).

The second form of interference, internal interference, occurs when the interfering factors belong to the theoretical domain of the generalization. In this regard, there is an important difference between psychology (as well as other human sciences) and the natural sciences. In the latter sciences, there are no in-principle obstacles to a gradual refinement of ceteris paribus clauses and to a formulation of laws that are exceptionless in their own domain, but there are good reasons to think that the same does not hold for laws involving intentional notions. Any generalization in terms of beliefs and desires is always threatened by the interference of competing beliefs and desires in a way that challenges any theoretical approach. Two arguments can be invoked in this context. In the first place, and assuming as true a holistic account of the psychological realm, the fact 
that the content of mental states is determined by their location in a network of propositional attitudes and that different interpretations may determine differently the content of beliefs, desires or intentions makes it impossible to fix all the variables involved in intentional explanations. In the second place, John McDowell's reflections on the uncodifiability of human rationality and, in particular, his thesis that human actions cannot be explained in terms of deductions from a definite set of universal principles also undermine the possibility of explaining human action on the basis of a nomological system. ${ }^{7}$ The uncodifiability thesis is quite plausible in the light of the serious difficulties that we face when we try to apply abstract, universal principles to concrete, particular cases. On the one hand, the vagueness and generality of principles contrast with the uncontrollable diversity of particular situations that defies any set of abstract rules. As a result, it is often possible to arrive at different conclusions departing from the same principle. On the other hand, there are also cases where equally valuable principles may contradict one another. Now, if an agent cannot act on the basis of a precise set of universal principles, the psychologist or sociologist cannot, a fortiori, explain her action in the form of deductions from universal principles or laws. As a result, generalizations couched in an intentional vocabulary do not support accurate predictions.

We can now raise the following question: does the explanation of human action involve ceteris paribus laws? It is not easy to answer this question, because putative ceteris paribus laws have, in the human sciences, an indefinite status; they are a borderline case between, on the one hand, non-scientific rules of thumb or rough generalizations, useful in our everyday practices, and, on the other hand, the laws of the natural sciences.

We could say that the concept of law is a "family-resemblance" concept in the sense that it identifies different kinds of lawlike generalizations without being able to reduce them to a well-defined set of defining characteristics. First, there are exceptionless laws. Some argue, as we have seen, that even the most basic laws of physics are not exceptionless; for instance, magnetic forces may create exceptions to the law of gravitation. However, and since apparent excep-

${ }^{7}$ See also Child 1993. 
tions to physical laws may be explained away with the help of other physical laws, we may consider the fundamental laws of physics as exceptionless. Second, there are the non-strict laws of the natural special sciences. Third, there are laws of the human special sciences which are not couched in an intentional vocabulary. They are vulnerable to forms of interference that are typical of the human sciences; for instance, ethical or cultural norms may interfere with well-established economic laws. Fourth, there are generalizations couched in an intentional vocabulary; they are such a pale echo of the basic laws of physics that it becomes disputable to call them laws. Their exceptions are ineliminable because there is not a theory able to deal with the effects of internal interference.

\section{4}

Now we must address a further problem: by claiming that the status of scientific generalizations is not the same in the natural and in the human sciences, are we not contributing to a devaluation of the human sciences and returning to an old-fashioned methodological dualism in the realm of science? The answer, as we shall see, is no.

According to the position defended in this article, the explanation of human action is based to a large extent on rationality assumptions. When we want to understand an action, we want typically to understand the reasons that motivated the action, and a mere reference to behavioural regularities is not enough. Human actions are, in most cases, the result of deliberations. It would be quite odd for an agent to take decisions on the basis of her previous behaviour and respective regularities; for the same reason, the explanation of human action cannot abstract totally from the deliberative process that originated a particular action. If our decisions and our self-understanding require the adoption of a deliberator's stance, we cannot explain satisfactorily the human action relying only on observable regularities. $^{8}$

However, one must also recognize that generalizations play an important role in the human sciences. In opposition to strict naturalistic perspectives that downplay the role of rationality assumptions

${ }^{8}$ For an elaboration of this point, see Kim 1998. 
in the explanation of human action and hermeneutic approaches that neglect the role of generalizations, we should attempt to articulate rationality assumptions and generalizations in the human sciences.

A prominent supporter of the so-called "simulation theory" in philosophy of mind and cognitive science, Robert Gordon, offers us a good example of such an articulation. The simulation theory is an account of our folk-psychological ability to explain and predict an agent's behaviour on the basis of an intentional vocabulary that includes notions like 'belief', 'desire' or 'intention'. According to a particular view of folk psychology, the so-called "theory-theory", folk psychology is a protoscientific theory, to the extent that it relies on generalizations that, by establishing correlations between thoughts and actions, allow us to predict human behaviour. Simulationists dispute the idea that our folk-psychological ability to explain, interpret and predict human behaviour is based on theoretical knowledge. Several objections may be addressed to the theory-theory. For instance, it is often argued that it is implausible to attribute the relevant theoretical knowledge to small children who are quite good at explaining and predicting actions. The idea that the explanation of human action follows universally shared principles is also controversial. For our purposes, however, the most significant objection against theory theory is based on the vagueness of the putative laws of folk psychology. As Goldman (1995) and Gordon (1995) point out, the laws of folk psychology are condemned to be vague because of the presence of ceteris paribus clauses. In fact, the number of interfering factors that can undermine predictions made on the basis of folk-psychological generalizations is so high that the explanatory relevance of such generalizations becomes questionable. As an alternative, simulation theorists claim that we should use ourselves as models for the interpretation of an agent; the proposal is to interpret other minds by projecting our emotions, motivations and deliberating processes.

In this context, Gordon's suggestion that the vagueness of ceteris paribus clauses cab be corrected through the method of empathy or simulation is particularly relevant. He rightly points out that by transposing ourselves into other people we can have access to reasons or factors that can interfere with established regularities, enabling us to explain and predict exceptions to reliable generalizations. 
How does one know how to recognize atypical situations or to expand the ceteris paribus clause? An answer is ready at hand. As long as one applies these generalizations in the context of practical simulation, the unspecifiable constraints on one's own practical reasoning would enable one to delimit the application of these rules. This gives one something to start with: as one learns more about others, of course, one learns how to modify these constraints in applying generalizations to them. (Gordon 1995: 67)

The same point can be made with the help of another distinguished supporter of simulation theory, Jane Heal (2003: 45-62). She rightly points out that the epistemic status of a belief depends on its relations with other, relevant beliefs. As a result, the interpretation of an agent requires sensitivity to the beliefs that an agent considers relevant to a given belief. But how can we identify the relevant beliefs? Certainly not by theory-building, because there is not a theory of relevance. In order to identify relevant beliefs, we have to rely on our own judgements of relevance and to interpret the agent on the basis of such judgements.

This appeal to simulation theory may seem to be incoherent with the Davidsonian premises that I presented above. Simulationists ground our mindreading abilities in psychological mechanisms, and a Davidsonian approach to the interpretation and explanation of human action is based, on the contrary, on rationality assumptions or in the famous Principle of Charity, according to which the interpretation of other agents must necessarily obey to presuppositions of truth and rationality.

However, the method of empathy or simulation is not incompatible with the Principle of Charity. In its initial formulations, the requirement of charity seemed to be a strong normative principle, based on an idealized assumption of truth and rationality in the interpretation of other people's utterances and actions. However, Davidson's later reflections on the Principle of Charity make it clear that the principle combines norms of rationality with considerations of psychological plausibility. The main point is not to maximize truth and rationality, but to avoid the attribution of unexplainable errors. Simulation may involve rationality assumptions to the extent that it reconstitutes the deliberative process of the interpretee; and charity is not a purely normative principle, because it can accommodate psychological intuitions. 


\section{References}

Cartwright, N. 1997. Where do laws of nature come from? Dialectica 51, 65-78. Cartwright, N. 2002. In favor of laws that are not ceteris paribus after all. Erkenntnis $57,425-439$.

Child, W. 1993. Anomalism, uncodifiability, and psychophysical relations. Philosophical Review 102, 215-245

Davidson, D. 1980. Essays on Actions and Events. Oxford: Clarendon Press.

Davidson, D. 1984. Inquiries into Truth and Interpretation. Oxford: Clarendon Press.

Davidson, D. 1995. Thinking causes. In Mental Causation, ed. by J. Heil and A. Mele. Oxford: Clarendon Press, 3-17.

Davidson, D. 2004. Problems of Rationality. Oxford: Clarendon Press.

Earman, J., and Roberts, J. 1999. Ceteris paribus, there is no problem of provisos. Synthese 118, 439-478.

Earman, J., Roberts, J. and Smith, S. 2002. Ceteris paribus lost. Erkenntnis 57, 281-301.

Fodor, J. 1987. Psychosemantics. Cambridge, MA: The MIT Press.

Fodor, J. 1991. You can fool some of the people all of the time, everything else being equal; Hedged laws and psychological explanations, Mind 100, 1991, 19-34.

Goldman, A. 1995. Interpretation psychologized. In Folk Psychology, ed. by M. Davies and T. Stone. Oxford: Blackwell, 74-99.

Gordon, R. 1995. Folk psychology as simulation. In Folk Psychology, ed. by M. Davies and T. Stone. Oxford: Blackwell, 60-73.

Heal, J., 2003. Mind, Reason, and Imagination: Selected Essays in Philosophy of Mind and Language. Cambridge: Cambridge University Press.

Hempel, C. 1988. Provisos: A problem concerning the inferential functions of scientific laws. In The Limits of Deductivism, ed. by A. Grünbaum and W. Salmon. Berkeley, CA: The University of California Press, 19-36.

Horgan, T. and Tienson, J. 1996. Connectionism and philosophy of psychology. Cambridge, MA: The MIT Press.

Kim, J. 1993. Supervenience and Mind. Cambridge: Cambridge University Press.

Kim, J. 1995. Can supervenience and 'non-strict laws' save anomalous monism? In Mental Causation, ed. by J. Heil and A. Mele. Oxford: Clarendon Press, $19-26$.

Kim, J. 1998. Reasons and the first person. In Human Action, Deliberation and Causation, ed. by J. Bransen and S. Cuypers. Dordrecht: Kluwer, 67-87.

Kincaid, J. 1996. Philosophical Foundations of the Social Sciences. Cambridge: Cambridge University Press.

Kincaid, J. 2004. There are laws in the social sciences. In Contemporary Debates in Philosophy of Science, ed. by C. Hitchcock. Oxford: Blackwell, 168-185.

Lange, M. 1993. Natural laws and the problem of provisos. Erkenntnis 38: 233 248 .

Lange, M. 2002. Who's affraid of ceteris-paribus laws? Or: How I learned to stop worrying and love them, Erkenntnis 57, 131-147.

McDowell, J. 1998. Mind, Value and Reality. Cambridge, MA: Harvard University

Press.
Pietroski, P. and Rey, G. 1995. When other things aren't equal: Saving ceteris paribus laws from vacuity. The British Journal for the Philosophy of Science 46, 
$81-110$

Roberts, J. 2004. There are no laws of the social sciences. In Contemporary Debates in Philosophy of Science, ed. by C. Hitchcock. Oxford: Blackwell, 151-167.

Schiffer, S. 1991. Ceteris paribus laws. Mind 100, 1-17.

Woodard, J. 2000. Explanation and invariance in the special sciences. The British Journal for the Philosophy of Science 51, 197-254.

Woodard, J. 2002. There is no such thing as a ceteris paribus law. Erkenntnis 57, 27-52. 